American Journal of Immunology 7 (3): 29-38, 2011

ISSN 1553-619X

(C) 2011 Science Publications

\title{
Interplay of Cytokines and Chemokines in the Pathogenesis of Systemic Lupus Erythematosus
}

\author{
${ }^{1}$ Dilip Shah, ${ }^{2}$ Ajay Wanchu and ${ }^{3}$ Archana Bhatnagar \\ ${ }^{1}$ Department of Dermatology, Massachusetts General Hospital, \\ Harvard Medical School, Massachusetts, United States of America, Boston \\ ${ }^{2}$ Division of Arthritis and Rheumatic Diseases-OP09, \\ Oregon Health and Science University, 3181 SW Sam Jackson, \\ Park Road, Portland, OR 97239, USA \\ ${ }^{3}$ Department of Biochemistry, Basic Medical Sciences Block, \\ Panjab University, Chandigarh, India
}

\begin{abstract}
Problem statement: Dysbalance of T-helper-cell (Th) cytokines and chemokines was suggested to contribute to the pathogenesis of Systemic Lupus Erythematosus (SLE). Recent reports suggest the involvement of cytokines and chemokines in the pathogenesis of SLE, but their relationship with each other and particularly in the severity of disease, was not yet understood. We analyzed the interaction between cytokines and chemokines and their relationship with severity of disease in SLE. Approach: Serum levels of cytokines and chemokines were determined by ELISA and severity of disease were measured by by using SLE Disease Activity Index (SLEDAI) score. Results: The serum levels of cytokine (IL-2, IFN- $\gamma$, IL-6, IL-10 and IL-12) and chemokine (CCL2, CCL5 and CXCL10) were variably associated with disease activity in SLE patients. Strict interaction between cytokines and chemokines was observed in SLE patients. Interleukin-6 was positively correlated with CCL5 while IL-12 was also analogous correlated with CXCL10 in SLE patients. The kidney involvement in SLE patients was related with intense increased levels of cytokines (IFN- $\gamma$, IL12) and chemokines (CCL2, CCL5 and CXCL10). Conclusion: These data suggest that interplay of cytokines and chemokines may be involved in the severity of disease. Also, a better understanding of the cytokines and chemokines interaction may likely to provide important clues to the pathogenic mechanism and pave the way toward more effective therapeutics.
\end{abstract}

Key words: Systemic Lupus Erythematosus (SLE), chronic autoimmune, population proliferates, chemoattractant molecules, American College of Rheumatology (ACR), levels upon correlating, cytokines present, secretes cytokines

\section{INTRODUCTION}

Systemic Lupus Erythematosus (SLE) is a chronic autoimmune disease characterized by the activation of $\mathrm{T}$ and polyclonal $\mathrm{B}$ lymphocytes, production of pathogenic autoantibodies and formation of immune complexes causing tissue and organ damage. The development of lupus and its manifestation involves several cell populations; however the $\mathrm{T}$ cells have been reported as a major contributor in the trigging and progression of disease (Cava, 2009). This heterogenous cell population proliferates differentially in response to antigen stimulation, secretes cytokines and triggers other cells to synthesize cytokines or chemokines (Kunz and Ibrahim, 2009), which are involved in the pathogenesis. The Interferon (IFN) $\gamma$ is known to induce synthesis of IL-12 by Antigen-Presenting Cell (APC) which generates IFN- $\gamma$ and IL-2 secreting Th1 cells. However, IL-4 induces T cells to develop into IL4, IL-5 secreting Th2 cells. Since the T cells with different cytokines profiles would lead to different subtypes, there has to be the right balance in their numbers. Hence, Th1/Th2 balance emerges as one of the known critical determinants for pathophysiology (Davis et al., 2011; Jacob and Stohl, 2011). There has been growing evidence suggesting that infiltration of $\mathrm{T}$

Corresponding Author: Archana Bhatnagar, Department of Biochemistry, Basic Medical Sciences Block, Panjab University, Chandigarh, India 
lymphocytes and other leucocytes into the sites of inflammation plays a critical role in organ involvement (Enghard et al., 2006). Leucocyte migration is mediated by the interaction of a number of chemokines and their receptors (Sallusto and Lanzavecchia, 2000). These chemoattractant molecules have well defined roles in directing cell movements necessary for the initiation of $\mathrm{T}$ cell immune response, attraction of appropriate effector cells to sites of inflammation and regulation of differential recruitment of $\mathrm{T}$ helper (Th1 and Th2) lymphocytes (Premack and Schall, 1996; Sallusto, 1999). Chemokines are known to play an important role in the development and progression of SLE (Eriksson $e t$ al., 2003). Increased levels of inflammatory chemokines in serum (Vila et al., 2007), plasma (Lit et al., 2006) and urine (Barbado et al., 2010) have been reported in SLE patients. The chemokine CCL 2 (Monocyte chemoattractant protein-1/MCP-1) is known as a Th1 chemokine and has an important role in homing of lymphoid cells to the zone of inflammation (Shimizu et al., 2005). Another chemokine CXCL 10 (Interferon-inducible protein-10/IP-10) upon binding to its ligand CXCR 3 attracts Th1 cells and promotes its maturation (Kong et al., 2009).

Although a number a cytokines and chemokines has been investigated as possible peacekeepers for the pathogenesis of SLE, specific correlation between cytokines and chemokines with each other have not been resolute yet and interplay of cytokine and chemokines with severity of disease and organ involvement is far to be identified. This study was designed to investigate the interplay of $\mathrm{T}$ cell cytokines, associated chemokines and disease severity which is required to improve disease prevention and diagnosis.

\section{MATERIALS AND METHODS}

Patients and controls: Patients for the study were selected from individuals attending out-patient Department of Internal Medicine at Postgraduate Institute of Medical Education and Research, Chandigarh, India. The study included 30 patients with SLE (28 females, 2 males) and the control group consisted of 30 healthy volunteers (28 females, 2 males). The SLE was diagnosed using the American College of Rheumatology (ACR) 1997 revised criteria (Hochberg, 1997) and disease activity was determined by using SLE Disease Activity Index (SLEDAI) score (Bombardier et al., 1992). The study protocol was approved by the Institute Ethics Committee,
Postgraduate Institute of Medical Education and Research, Chandigarh, India and informed consent was obtained from all the patients and controls (healthy subjects). The patients enrolled in the present study were non-smokers and non-alcoholics, not associated with any other autoimmune disease and were not undergoing any anti-inflammatory therapy.

Blood samples: Venous blood samples from patients and controls were collected into plain vacutainers (Becton Dickinson, USA), serum was separated, aliquoted and used for estimation of the cytokines (IL2 , IFN- $\gamma$, IL-4, IL-6, IL-10 and IL-12) and chemokines (CCL 2, CCL5 and CXCL 10).

Cytokines assay: The serum cytokine concentrations of SLE patients and control subjects were measured by Enzyme-Linked Immunosorbent Assay (ELISA) using OptEIA reagent kits (Pharmingen, BD Biosciences, San Diego, CA) according to the manufacturer's instructions. For each cytokine the specific kit was used wherein briefly, diluents provided was added, followed by addition of the sample and incubation at $37^{\circ} \mathrm{C}$ for 2 h. The wells were washed 3-4 times, the working detector (Enzyme labeled conjugated antibody) was added and incubated for $1 \mathrm{~h}$ at Room Temperature (RT). The substrate was added and kept in dark for $30 \mathrm{~min}$ at RT. Stop solution $(50 \mu \mathrm{L})$ was added at the end of incubation and the plate was read at $450 \mathrm{~nm}$.

Chemokines assay: The serum levels of CCL 2, CCL5 and CXCL 10 in all the samples were measured by Enzyme-Linked Immunosorbent Assay (ELISA) using OptEIA reagent sets (Pharmingen, BD Biosciences, San Diego, CA). Briefly, wells of the ELISA plate were coated with the capture antibody diluted in coating buffer and incubated at $4^{\circ} \mathrm{C}$ overnight. Plates were washed thrice with wash buffer and blocked with assay diluent for $1 \mathrm{~h}$ at RT. The standards and sample were prepared in assay diluent, added accordingly and incubated at RT for 2 $\mathrm{h}$. Washing thrice was carried out upon completion of this step and the biotinylated antibodies as well as the enzyme reagent (streptavidin-horse radish peroxidase) were added before incubating the plate for $1 \mathrm{~h}$ at RT. The plates were washed seven times before incubating with Tetramethylbenzidine and hydrogen peroxide for $30 \mathrm{~min}$ at RT in dark. The reaction was stopped with $2 \mathrm{~N} \mathrm{H}_{2} \mathrm{SO}_{4}$ and read at $450 \mathrm{~nm}$.

Statistical analysis: Statistically significant differences were determined using the Mann-Whitney $U$ test. Correlation analyses were performed using two-tailed 
Spearman's rank correlation. Analyses were performed using GraphPad Prism v.5.00.288 for Windows (GraphPad Software, San Diego, CA) p values less than 0.05 were considered significant.

\section{RESULTS}

Demographic profile of subjects: The mean age of patients with SLE was $25.8 \pm 6.50$ years and that of control group was $26.43 \pm 6.20$ years. The demographic and clinical characteristics of SLE patients and healthy controls are summarized in the Table 1. Most of the patients had been suffering from the disease for a couple of years. The disease activity determined by SLE Disease Activity Index (SLEDAI) score (maximum score of 105: Mild score <10; Moderate score 10-20; Severe score >20) showed three patients

with mild, five patients with moderate SLEDAI score while 22 patients were in the severe score category. The elevated level of laboratory parameters; sedimentation rate of the Erythrocytes (ESR), complement levels of $\mathrm{C} 3$ and C4 were measured in SLE patients which reflect severity of disease.

Table 1: Demographic characteristics of patients with Systemic Lupus Erythematosus (SLE) and healthy controls

\begin{tabular}{lll}
\hline & SLE patients & Controls \\
\hline Number $(\mathrm{n})$ & 30 & 30 \\
Female/male & $28 / 2$ & $28 / 2$ \\
Age $(\mathrm{yrs})$ & $25.8 \pm 6.50$ & $26.43 \pm 6.20$ \\
Duration of disease & $2.38 \pm 1.80$ & NA \\
ESR $(\mathrm{mm} / \mathrm{hr})$ & $48.35 \pm 24.50$ & NA \\
C3 $(\mathrm{mg} / \mathrm{dl})$ & $56.93 \pm 18.62$ & NA \\
C4 $(\mathrm{mg} / \mathrm{dl})$ & $27.39 \pm 12.934$ & NA \\
SLEDAI Score & $26.22 \pm 10.90$ & NA \\
\hline
\end{tabular}

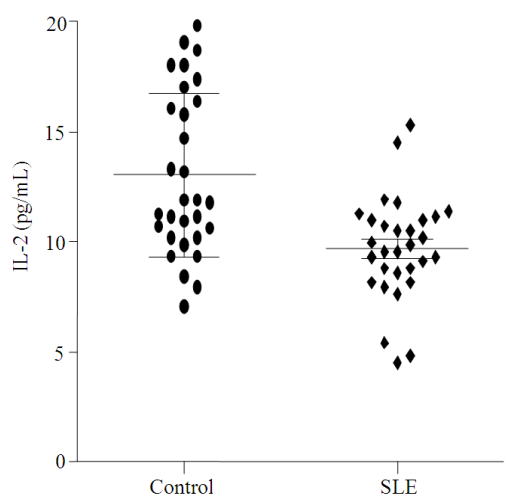

(a)

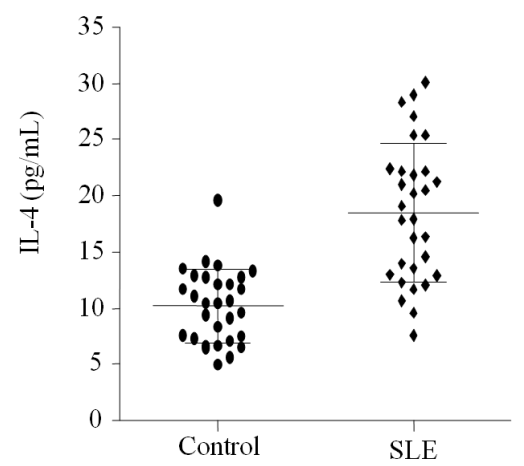

(d)

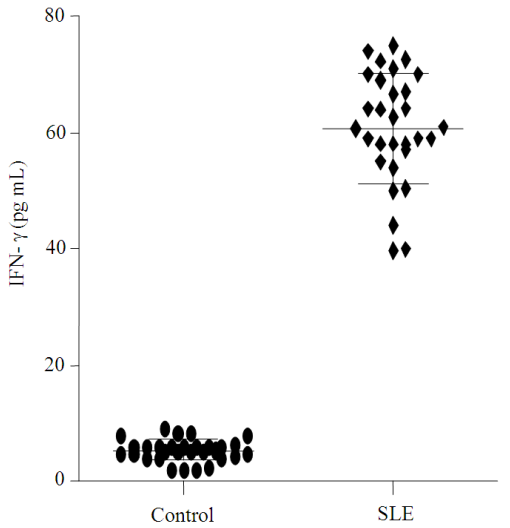

(b)

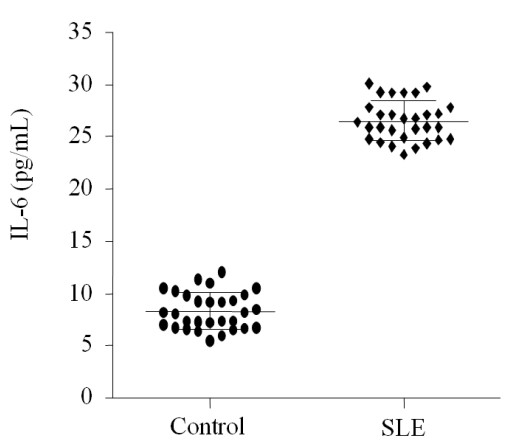

(e)

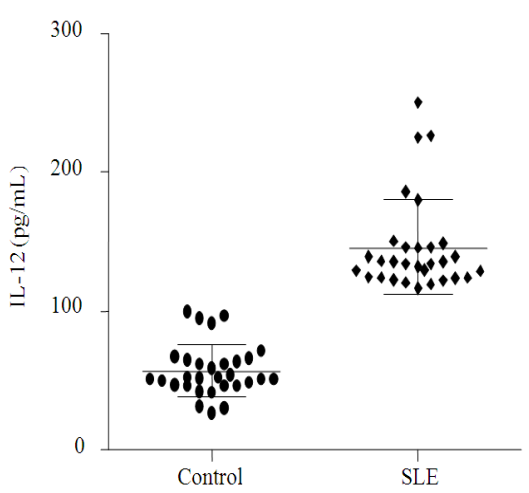

(c)

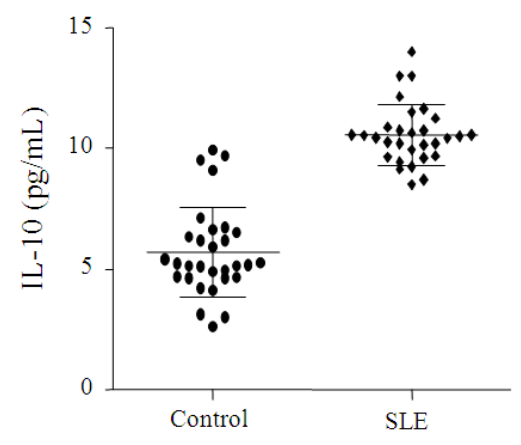

(f)

Fig. 1: Th1/Th2 profile in healthy controls and SLE patients: a) Serum levels of IL-2 (pg/mL) determined by ELISA in samples from healthy controls and SLE patients. (b) serum levels of IFN- $\gamma(\mathrm{pg} / \mathrm{mL})$ in sera samples of controls and SLE patients. c) serum levels of $\mathrm{IL}=12(\mathrm{pg} / \mathrm{mL})$ in sera samples of controls and SLE patients. (d) serum levels of IL-4 (pg/mL) in healthy controls and SLE patients. (e) serum levels of IL$6(\mathrm{pg} / \mathrm{mL})$ in healthy controls and SLE patients. f) serum IL-10 levels (pg/mL) in healthy controls and SLE patients. Results are expressed as mean \pm S.D.; $* \mathrm{p}<0.05$, $* * \mathrm{p}<0.01$ and $* * * \mathrm{p}<0.001$ when compared to healthy controls 
Am. J. Immunol., 7 (3): 29-38, 2011

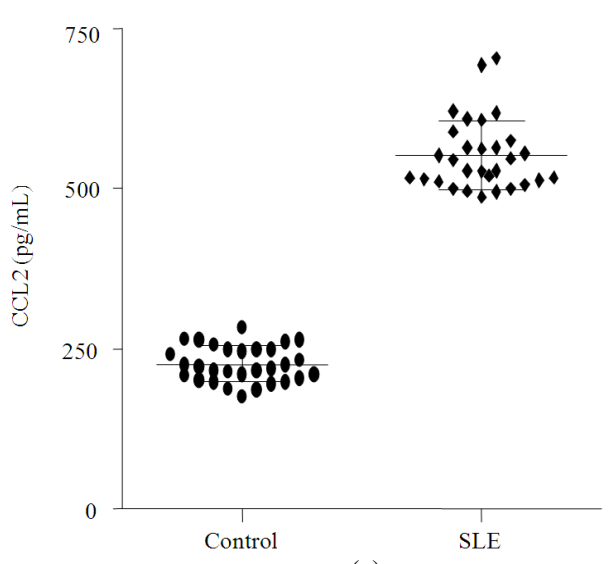

(a)

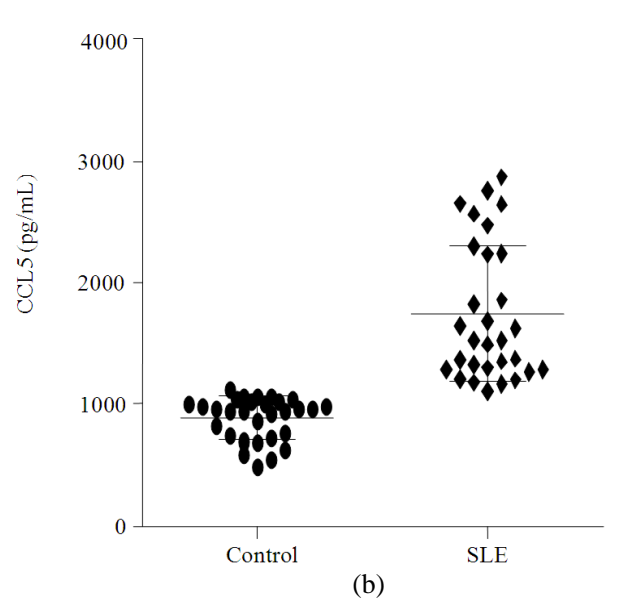

(b)

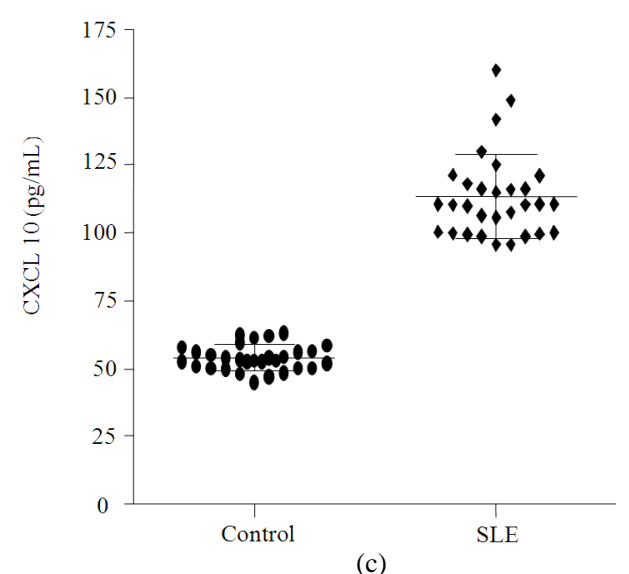

(c)

Fig. 2: Chemokine levels in healthy controls and SLE patients: (a) serum levels of CCL $2(\mathrm{pg} / \mathrm{mL})$ in healthy controls and SLE patients. (b) serum levels of CCL5 $(\mathrm{pg} / \mathrm{mL})$ in healthy controls and SLE patients. (c) Levels of CXCL 10 (pg/mL) in sera of healthy controls and SLE patients. Results are expressed as mean \pm S.D.; ${ }^{*} p<0.001$ significantly differ from healthy controls
Th1/Th2 cytokine profile: Serum levels of the Th1/Th2 cytokine are depicted in Fig. 1. The levels of IL-2 was significantly $(\mathrm{p}<0.001)$ decreased $(9.67 \pm 2.36$ pg $\mathrm{mL}^{-1}$ ) in SLE patients as compared to healthy controls $\left(13.03 \pm 3.69 \mathrm{pg} \mathrm{mL}^{-1}\right)$ (Fig. 1a). The levels of pro-inflammatory cytokine IFN- $\gamma$ was notably $(\mathrm{p}<0.001)$ increased in SLE patients $(60.86 \pm 9.48 \mathrm{pg}$ $\left.\mathrm{mL}^{-1}\right)$ as compared to healthy controls $(5.42 \pm 1.86 \mathrm{pg}$ $\mathrm{mL}^{-1}$ ). A key cytokine for $\mathrm{T}$ cell differentiation; IL-12 has an important role in autoimmune disorders. The SLE patient samples exhibited substantially higher values of the cytokine $\left(57.23 \pm 18.65 \mathrm{pg} \mathrm{mL}^{-1}\right)$ as compared to the healthy controls $\left(145.9 \pm 34.0 \mathrm{pg} \mathrm{mL}^{-1}\right)$. The order of significance found was that of $p<0.001$ (Fig. 1c). As compared to controls $(10.19 \pm 3.20 \mathrm{pg}$ $\left.\mathrm{mL}^{-1}\right)$, a significant $(\mathrm{p}<0.05)$ but variable increase in IL-4 levels of serum from SLE patients $(18.54 \pm 6.17 \mathrm{pg}$ $\mathrm{mL}^{-1}$ ) was observed (Fig. 1d). The levels of IL-6 (Fig. 1e) were markedly elevated $(p<0.001)$ in SLE patients $\left(26.52 \pm 1.89 \mathrm{pg} \mathrm{mL}^{-1}\right)$ as compared to controls $\left(8.33 \pm 1.75 \mathrm{pg} \mathrm{mL}^{-1}\right)$. As shown in Fig. 1f, the levels of IL-10 were higher $(\mathrm{p}<0.001)$ in SLE patients $\left(10.58 \pm 1.24 \mathrm{pg} \mathrm{mL}^{-1}\right)$ as compared to healthy individuals $\left(5.72 \pm 1.86 \mathrm{pg} \mathrm{mL}^{-1}\right)$.

Chemokine profile: The levels of chemokine are portrayed in Fig. 2. The levels of chemotactic chemokine CCL2 was considerably $(\mathrm{p}<0.001)$ increased in SLE patients $\left(552.0 \pm 55.27 \mathrm{pg} \mathrm{mL}^{-1}\right)$ as compared to healthy controls $\left(226 \pm 28.08 \mathrm{pg} \mathrm{mL}^{-1}\right)$. As depicted in Fig. 2b, the levels of CCL5 in SLE (1742 \pm $\left.563.4 \mathrm{pg} \mathrm{mL}^{-1}\right)$ was remarkably $(\mathrm{p}<0.001)$ elevated as compared to healthy controls $\left(889.8 \pm 174.8 \mathrm{pg} \mathrm{mL}^{-1}\right)$. Similar pattern of increase $(\mathrm{p}<0.001)$ in the levels of inflammatory chemokine CXCL10 were obtained in SLE patients $\left(113.4 \pm 15.50 \mathrm{pg} \mathrm{mL}^{-1}\right)$ and healthy controls $\left(53.96 \pm 4.69 \mathrm{pg} \mathrm{mL}^{-1}\right)$.

Th1/Th2 cytokines and chemokines with each other and also with the severity of disease, correlations studied were accomplished. The values of spearman's correlation coefficients were shown in Fig. 3 and 4. A negative correlation $(\mathrm{r}=-0.473, \mathrm{p}<0.01)$ was observed between serum IL-2 and SLEDAI score (Fig. 3a). Notably a positive correlation $(\mathrm{r}=0.532, \mathrm{p}<0.01)$ was observed between IFN- $\gamma$ and SLEDAI score in SLE patients (Fig. 3b). As shown in Fig. 3b, the levels IL-12 was substantially positively $(r=0.371, \quad \mathrm{p}<0.05)$ associated with the disease activity measure as SLEDAI score. An affirmative association $(\mathrm{r}=0.532, \mathrm{p}<0.01)$ was existed between IL-6 and SLEDAI score (Fig. 3d). 


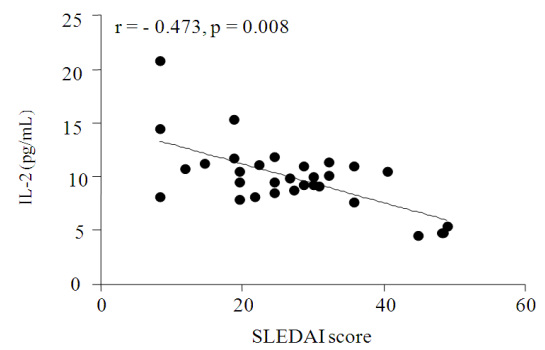

(a)

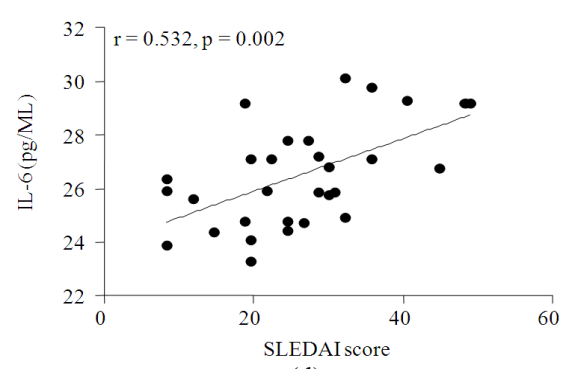

(d)

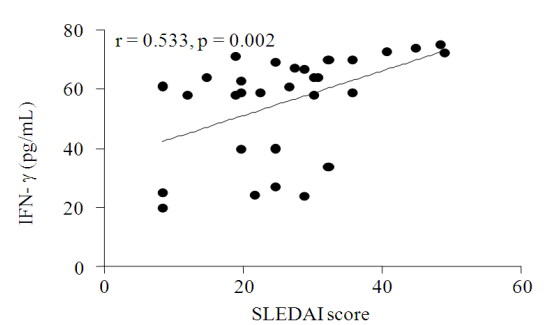

(b)

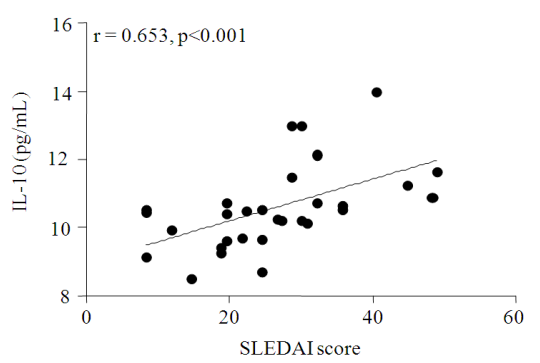

(e)

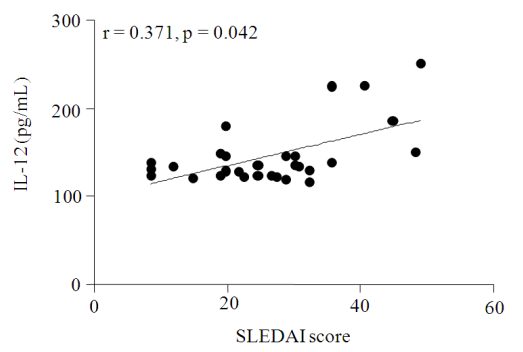

(c)

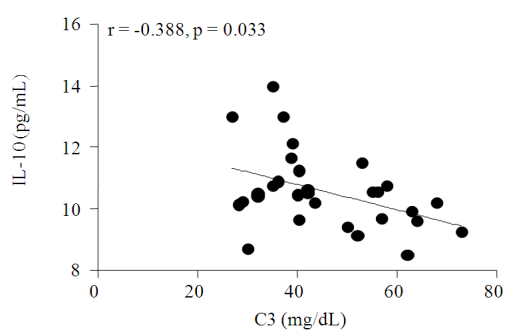

(f)

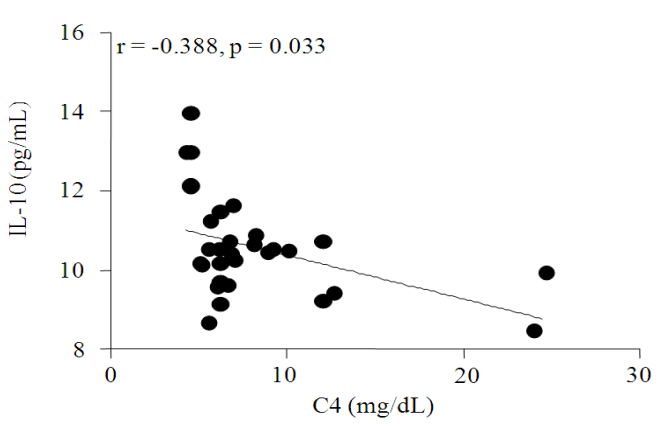

(g)

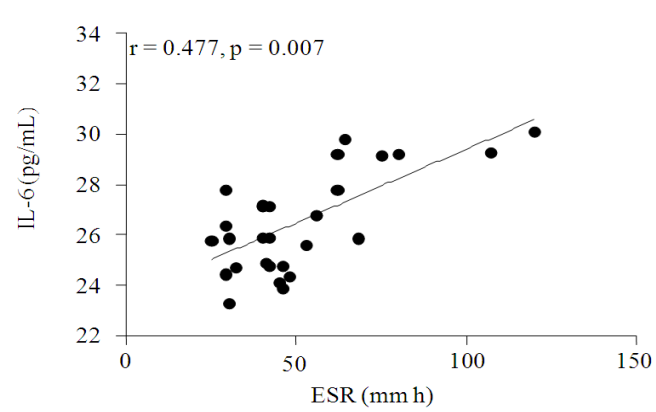

(h)

Fig. 3: Correlation amongst cytokines, SLEDAI score, Erythrocyte Sedimentation coefficient Rate (ESR) and complement proteins (C3 and C4) in SLE patients where $r=$ Spearman's rank correlation coefficient and $p$ is level of significance. (a) relationship between IL-2 levels and SLEDAI score $(r=-0.473, p<0.01)$. (b) relationship between IFN- $\gamma$ levels and SLEDAI score $(r=0.533, p<0.01)$. (c) relationship between IL-12 level and SLEDAI score $(\mathrm{r}=0.371, \mathrm{p}<0.05)$. (d) relationship between IL-6 level and SLEDAI score $(\mathrm{r}=$ $0.532, \mathrm{p}<0.01)$. e) relationship between IL-10 level and SLEDAI score $(\mathrm{r}=0.653, \mathrm{p}<0.001)$. (f) relationship between IL-10 level and C3 $(r=-0.388, \mathrm{p}<0.05)$. (g) relationship between IL-10 level and C4 $(\mathrm{r}=-0.388$, $\mathrm{p}<0.05)$. h) relationship between IL- 6 and ESR $(r=-0.477, \mathrm{p}<0.01)$

Correlation studies: To evaluate the relationship of As shown in Fig. 3e, a clear-cut relation $(r=0.563 p<0.001)$ was found between IL-10 and SLEDAI score in SLE patients. A significant correlation was observed between IL-10 and clinical parameters; $\mathrm{C} 3(\mathrm{r}=-0.388 ; \mathrm{p}<0.05)$ and $\mathrm{C} 4(\mathrm{r}=-0.388 ; \mathrm{p}<0.05)$ as depicted in Fig. $3 \mathrm{f}$ and $3 \mathrm{~g}$. The IL-6 levels upon correlating with ESR revealed a considerable positive association $(r=0.477, \mathrm{p}<0.01)$ in SLE patients (Fig. 3h).

Furthermore, the serum chemokines CCL 2, CCL 5 and CXCL 10 showed a major positive correlation with disease activity measured as SLEDAI score in SLE patients $(r=0.488, p<0.01$, Fig. $4 a),(r=$ $0.557, \mathrm{p}<0.01$, Fig. $4 b)$ and $(r=0.533$, p<0.01, Fig. $4 c)$ respectively. Interaction between cytokines and chemokines has been observed in SLE patients in the present study.

The levels of CCL 5 showed a remarkable positive correlation $(r=0.465, p<0.01$, Fig. $4 d)$ with serum IL-6 in SLE patients. Also, CXCL 10 levels correlated positively with IL-12 $(r=0.368, p<0.05$, Fig. $4 \mathrm{e})$ and IFN- $\gamma(\mathrm{r}=0.533, \mathrm{p}<0.01$, Fig. $4 \mathrm{f})$ in SLE patients. 
Am. J. Immunol., 7 (3): 29-38, 2011

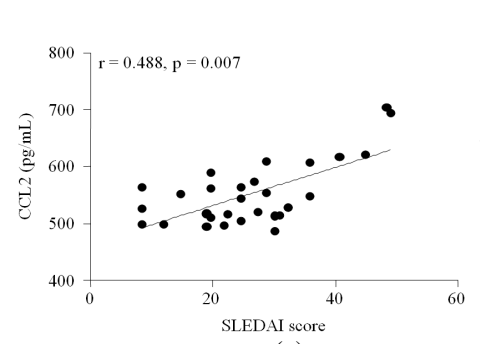

(a)

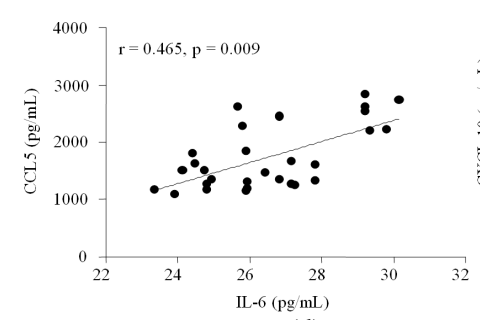

(d)

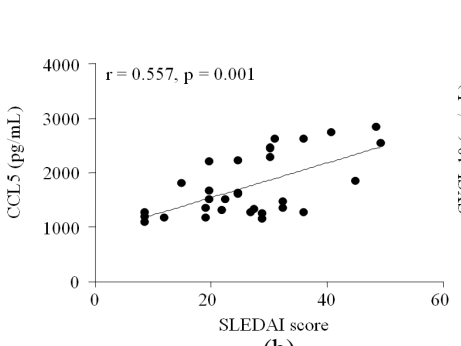

(b)

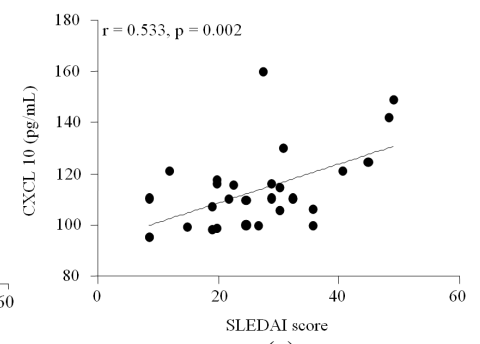

(c)

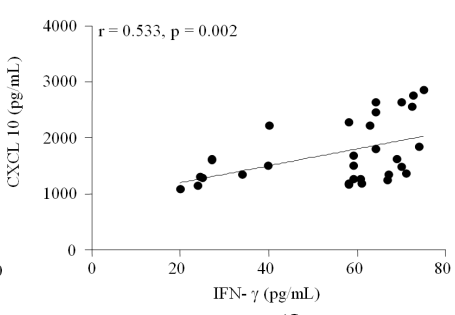

(f)

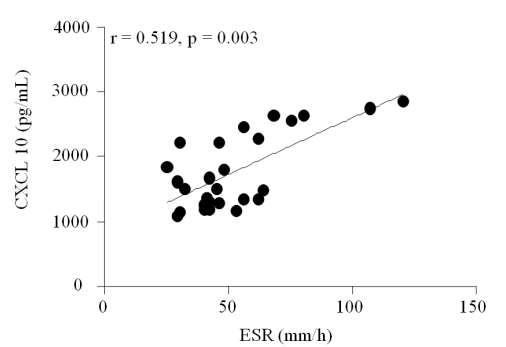

(g)

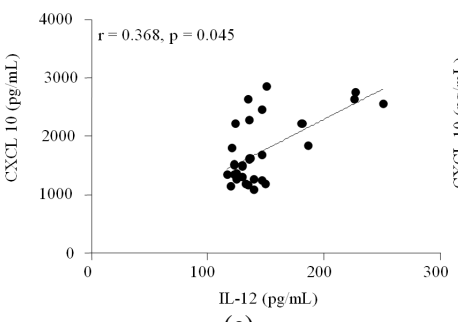

(e)

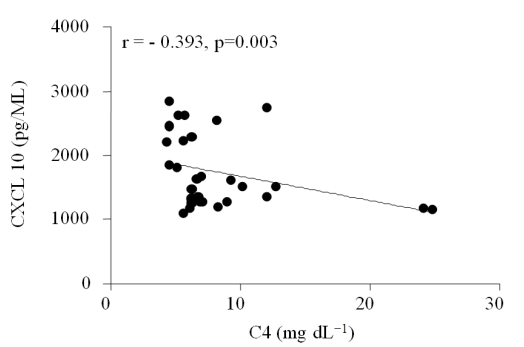

(h)

Fig. 4: Correlation of chemokines with SLEDAI score, ESR and IL-12 in SLE patients revealed the following associations: (a) relationship between CCL 2 and SLEDAI score $(r=0.488, p<0.01)$. (b) relationship between CCL 5 and SLEDAI score $(r=0.557, \mathrm{p}<0.01)$. (c) relationship between CXCL 10 and SLEDAI score $(\mathrm{r}=0.533, \mathrm{p}<0.01)$. (d) relationship between CCL 5 and IL-6 $(\mathrm{r}=0.465, \mathrm{p}<0.01)$. (e) relationship between CXCL 10 and IL-12 $(r=0.368, p<0.05)$. f) relationship between CXCL 10 and IFN- $\gamma(r=0.533$, $\mathrm{p}<0.01)$. (g) relationship between CXCL 10 and ESR $(r=0.519, \mathrm{p}<0.01) . \quad \mathrm{h})$ relationship between CXCL 10 and $\mathrm{C} 4(\mathrm{r}=-0.393, \mathrm{p}<0.05)$

Moreover, the levels of CXCL 10 showed incredibly positive correlation with ESR $(r=0.519$, $p<0.01$, Fig. 4 g) and $\mathrm{C} 4(\mathrm{r}=-0.393$, p $<0.05$, Fig. $4 \mathrm{~h})$ in SLE patients. No such correlations were observed in healthy controls. In the present study, the major clinical problem had seen as obsessive association of nephritis with SLE patients. Out of 30 patients with SLE, 6 patients with increased levels of cytokines; IL-12 and IFN- $\gamma$ and chemokines; CCL-2 and CCL5 had involvement of kidney (nephritis). These patients had also low level of laboratory parameter like C3 and C4.

\section{DISCUSSION}

There is growing evidence that cytokines and chemokines playing vital roles in the initiation and continuation of autoimmune disorders such as SLE. A defective regulation of Th1 and Th2 cytokines has been found typically in SLE and a Th1/Th2 imbalance appears to be an important factor responsible for the pathophysiology in lupus nephritis (Cava, 2009). The cytokine imbalance can thereby lead to alter immune responses which might influence the chemokines and subsequent severity of the disease. The present study evaluates the relationship between Th1- Th2 cytokine levels with the Th1 or Th2 chemoattractants chemokines and SLEDAI score of SLE patients. IL-2, a key molecule, known to maintain a link between autoimmune disorders such as SLE and alterations in the immunity \& tolerance is a signature cytokine of Th1 cells. Deficient IL-2 synthesis has been suggested to have a physiopathogenic role in autoimmunity (Gomez- 
Martin et al., 2009). However, murine models of mice and SLE in humans are known to show reduced IL-2 synthesis similar to our observations (Gomez-Martin et al., 2009).

The other Th1 cytokine IFN- $\gamma$ was higher, which can be attributed to elevated IL-12 levels as shown by (Tokano et al., 1999). Santer et al. (2009) demonstrated that enhanced IFN- $\gamma$ production leads to the tissue damage responsible for clinical symptoms in patients with SLE (Santer et al., 2009). In the previous study, we have showed that elevate levels of IFN $-\gamma$ in the culture supernatant from SLE patients were associated with nephritis (Shah et al., 2010). In the current study, a positive correlation between serum IFN- $\gamma$ and SLEDAI score suggests that IFN- $\gamma$ is actively involved in the pathogenesis of the disease. Promotion of IFN- $\gamma$ production by IL-12 has been reported to lead to polarization of peripheral cells to Th1 phenotype in lupus nephritis (Tucci et al., 2008). They have reported that intrarenal inflammation and cytokine imbalance of peripheral cells toward a Th1 phenotype in lupus nephritis. The renal damage has been suggested to be promoted by the imbalance of the peripheral IFN- $\gamma$ : IL4. It was shown that $\mathrm{T}$ cells from lupus patients tilted to a Th1 phenotype with low IL-2 and a high IFN- $\gamma$ expression toning the severity of lupus. Also, the IFN- $\gamma$ correlated positively with the SLEDAI score.

The elevated level of cytokine IL-4 observed in patients in our study was contrary to the previous reports of Funauchi et al. (1991) and Shiroiwa et al. (2007). However, our results are similar to the study of Wong et al. (2000). Shiroiwa group has suggested that the role of IL-4 in the pathogenesis may differ between certain subsets of SLE, even if they show similar disease phenotypes. Several lines of evidence suggest that IL-6 plays a central role in the pathogenesis of SLE; including regulation of growth and differentiation of B cells and auto-antibody production and that it may have a direct role in mediating tissue damage (Youinou and Jamin, 2009). In our study, serum IL-6 was found to be higher in lupus nephritis patients $(n=6)$ and it also positively correlated with ESR and SLEDAI score. The result is similar to the study of (Chun et al., 2007) and (Esposito et al., 2009) who advocate that this cytokine is like a biomarker which moves up and down with inflammation and treatment respectively. Reports from other laboratories suggest its role in manifestation of neuropsychiatric SLE along with other cytokines such as IL-2, IFN- $\gamma$ and IL-10 (Efthimiou and Blanco, 2009).

Interleukin-10, a B-cell stimulatory cytokine is known to inhibit type 1 cytokine response and has a critical role in the immunopathogenesis of SLE
(Rousset et al., 1992). Spontaneous and mitogeninduced IL-10 levels have been reported to be higher in SLE patients (Llorente et al., 1994). The levels of IL-10 were remarkably higher in SLE patients as compared to healthy controls. Furthermore, a positive correlation of serum IL-10 levels with disease activity and a negative correlation of this cytokine with complement proteins $\mathrm{C} 3$ and C4 have been observed in SLE patients which is very similar to other studies (Chun et al., 2007; Park et al.,1998). It has been reported that monocytes and $\mathrm{CD}^{+}$as well as $\mathrm{CD}^{+} \mathrm{T}$ lymphocytes contribute to overproduction of IL-6 and IL-10 in SLE (Mellor-Pita et al., 2009) which correlated with the disease activity but was independent of the treatment the patients received. The role of cytokine balances in classification of renal pathology has been shown to involve in laser micro dissection of renal biopsy (Wang et al., 2010). Interleukin-12, a proinflammatory cytokine induces the production of IFN- $\gamma$, favors the differentiation of Th1 cells and links innate resistance and adaptive immunity (Bertagnolli et al., 1992). There are conflicting differences in the reports on IL-12 activity in SLE: It was reported that IL-12 production by PBMC was lower in SLE patients than in healthy controls (Liu and Jones 1998), whereas plasma level of IL-12 in active SLE patients was found to be higher than in the controls (Wong et al., 2000). The present study, levels of serum IL-12 were high and positive correlation with SLEDAI score in SLE patients. Higher serum IL-12 levels have been found to be indicative of nephritis (Tucci et al., 2008) in the present study. This discrepancy in the result may be due to diverse manifestation of SLE.

Recent studies have shown that chemokines and their receptors are intimately involved in regulating organ specific leucocyte trafficking and inflammation, suggesting their important roles in the pathophysiology of SLE (Vielhauer et al., 2007). These observation added the association between cytokines and chemokines in the leucocyte trafficking and inflammation in SLE. The chemokine monocyte chemotactic protein 1 (CCL2) is mainly expressed by activated monocyte/macrophages, helper T1 (Th1) cells and natural killer cells and it attracts leucocytes and other mediators into sites of inflammation (Lit et al., 2006). The chemoattractants for Th1 lymphocytes; CCL5 and CXCL10 are synthesis and expression from neutrophils, macrophages and other immune cells is induced by IFN- $\gamma$ and is suppressed by IL-10 and IL-4 in autoimmune disease (Zlotnik and Yoshie, 2000). In the present study, the levels of inflammatory chemotactic chemokines for Th1 and Th2 cells (CCL2, CCL5 and CXCL 10) were significantly higher in SLE patients and positively correlated with disease activity. 
Patients $(n=6)$ with increased levels of cytokines; IL-12 and IFN- $\gamma$ and chemokines; CCL-2 and CCL5 had involvement of kidney (nephritis). Our result is restricted with the study of (Lit et al., 2006) and (Bauer et al., 2009). They have showed that CCL-2 and CXCL 10 were higher in SLE patients and plasma IL-18 concentration showed strong positive correlation with inflammatory chemokines; CXCL 10 and may be better indicators to assess the current disease activity, prediction of future disease flares and overall clinical decision-making. Our report shows elevated CXCL 10 levels in sera from SLE patients who could serve as good indicators of severity and activity of disease, as suggested by studies conducted at cellular level (Kong et al., 2009). Also, a good association between CXCL 10 and ESR in SLE may suggest Th1 chemokine activity in the disease which may have important roles in leukocyte homing, in the perpetuation of inflammation and concomitant tissue damage (Lee $e t$ al., 2009). Parallel to the positive correlation of plasma IL-18 with IP-10 (Bauer et al., 2009), in the present study, strong positive correlations were obtained between some of the cytokines and chemokines; the level of IFN- $\gamma$ was positively correlated with CXCL 10, the level of IL- 6 was positively associated with CCL 5 and the level of IL-12 was also positively linked with the level of CXCL 10 in SLE patients. These correlation may suggests that increase level of IFN- $\gamma$ may be due to excessive accumulation of neutrophils at the inflammatory site and increased production of CXCL 10 in SLE patients (Gasperini et al., 1999). Also, a good association of CXCL 10 with C4 and ESR in SLE patients in the present study may suggest that Th1 chemokine activity in the disease which may have important roles in leukocyte homing, in the perpetuation of inflammation and concomitant tissue damage (Lee et al., 2009).

The leucocytes at the sites of acute inflammation depend on the repertoire of inducible chemokines synthesized locally, which are, in part, influenced by pro-inflammatory cytokines present in the local environment of the cells at the time of stimulation (Lit et al., 2006). The synthesis and expression of Th1 chemoattractants, CCL5 and CXCL10 from neutrophils, macrophages and other immune cells is induced by IFN $\gamma$ and this response is suppressed by IL10 and IL4 (Gasperini et al., 1999). These reports may support our finding that cytokine and chemokine are interdepending and involved in the pathogenesis of SLE. Additional, the inflammatory cytokines may even enhance the Th1 mediated inflammatory process, the activation and migration of leukocytes and macrophages for initiation and perpetuating the inflammatory chemokines production.

\section{CONCLUSION}

In conclusion, present study depicts that imbalance Th1-Th2 cytokines and chemokines may be involved in the auto-inflammatory process of SLE. Taken together with present finding on the association of cytokines (IFN- $\gamma$, IL-6, IL-10 and IL-12) and inflammatory chemokine (CCL5 and CXCL 10), the result may provide new clue for their potential roles in the exacerbation of SLE disease and may act as a new reliable marker for SLE. However, more intensive studied are required to clarify the possible role of inflammatory chemokines and their association with cytokines involved in the inflammatory mechanismincluding cell activation, tissue destruction and other process underlying the immunepathogenesis of SLE.

\section{ACKNOWLEDGEMENT}

The reaches would like to acknowledge Dr. Aman Sharma for analyzing the clinical details of the patients.

Declaration of interest: The authors report no conflicts of interest.

\section{REFERENCES}

Barbado, J., L. Vega, R. Gonzalez-Gallego, A. Jimeno and R.O.D. Lejarazu et al., 2010 MCP-1 in urine as biomarker of renal lupus in absence of cytokines, interferon- $\gamma$ and growth factors. Reumatol Clin., 6: 296-298. PMID: 21794736

Bauer, J.W., M. Petri, F.M. Batliwalla, T. Koeuth and J. Wilson et al., 2009. Interferon-regulated chemokines as biomarkers of systemic lupus erythematosus disease activity: A validation study. Arthritis Rheum., 60: 3098-3107. PMID: 19790071

Bertagnolli, M.M., B.Y. Lin, D. Young and S.H. Herrmann, 1992. IL-12 augments antigendependent proliferation of activated $\mathrm{T}$ lymphocytes. J. Immunol., 149: 3778-3783. PMID: 1360994

Bombardier, C., D.D. Gladman, M.B. Urowitz, D. Caron and C.H. Chang, 1992. Derivation of the SLEDAI. A disease activity index for lupus patients. The committee on prognosis studies in SLE. Arthritis Rheum., 35: 630-640. PMID: 1599520

Cava, A.L., 2009. Lupus and T cells. Lupus, 18: 196201. DOI: $10.1177 / 0961203308098191$ 
Chun, H.Y., J.W. Chung, H.A. Kim, J.M. Yun and J.Y. Jeon et al., 2007. Cytokine IL-6 and IL-10 as biomarkers in systemic lupus erythematosus. J. Clin. Immunol., 27: 461-166. PMID: 17587156

Davis, L.S., J. Hutcheson and C. Mohan, 2011. The Role of cytokines in the pathogenesis and treatment of systemic lupus erythematosus. J. Interferon Cytokine Res, 31: 781-789. PMID: 21787222

Efthimiou, P. and M. Blanco, 2009. Pathogenesis of neuropsychiatric systemic lupus erythematosus and potential biomarkers. Mod. Rheum., 19: 457-468. PMID: 19618249

Enghard, P., D. Langnickel and G. Riemekasten, 2006. $\mathrm{T}$ cell cytokine imbalance towards production of IFN-gamma and IL-10 in NZB/W F1 lupus-prone mice is associated with autoantibody levels and nephritis. Scand J. Rheumatol, 35: 209-216. PMID: 16766368

Eriksson, C., K. Eneslatt, J. Ivanoff, S. RantapaaDahlqvist and K.G. Sundqvist, 2003. Abnormal expression of chemokine receptors on T-cells from patients with systemic lupus erythematosus. Lupus. 12: 766-774. PMID: 14596426

Esposito, P., M.M. Balletta, A. Procino, L. Postiglione and B. Memoli, 2009. Interleukin-6 release from peripheral mononuclear cells is associated to disease activity and treatment response in patients with lupus nephritis. Lupus, 18: 1329-1330. PMID: 19850661

Funauchi, M., M. Minoda and A. Horiuchi, 1991. Relationship between serum interleukin-4 or interferon-gamma level and B cell abnormality in patients with collagen vascular diseases. Ryumachi, 31: 493-501. PMID: 1767343

Gasperini, S., M. Marchi, F. Calzetti, C. Laudanna and L. Vicentini et al., 1999. Gene expression and production of the monokine induced by IFNgamma (MIG), IFN-inducible $\mathrm{T}$ cell alpha chemoattractant (I-TAC) and IFN-gammainducible protein-10 (IP-10) chemokines by human neutrophils. J. Immunol., 162: 4928-4937. PMID: 10202039

Gomez-Martin, D., M. Diaz-Zamudio, J.C. Crispin and J. Alcocer-Varela, 2009. Interleukin 2 and systemic lupus erythematosus: Beyond the transcriptional regulatory net abnormalities. Autoimmun Rev., 9: 34-39. PMID: 19269352

Hochberg, M.C., 1997. Updating the American College of Rheumatology revised criteria for the classification of systemic lupus erythematosus. Arthritis Rheum., 40: 1725-1725. PMID: 9324032

Jacob, N. and W. Stohl, 2011. Cytokine disturbances in systemic lupus erythematosus. Arthritis. Res. Ther., 13: 228-228. PMID: 21745419
Kong, K.O., A.W. Tan, B.Y. Thong, T.Y. Lian and Y.K. Cheng et al., 2009. Enhanced expression of interferon-inducible protein-10 correlates with disease activity and clinical manifestations in systemic lupus erythematosus. Clin. Exp. Immunol., 156: 134-140. PMID: 19175619

Kunz, M. and S.M. Ibrahim, 2009. Cytokines and cytokine profiles in human autoimmune diseases and animal models of autoimmunity. Mediators Inflamm., 2009: 979258-979258. PMID: 19884985

Lee, E.Y., Z.H. Lee and Y.W. Song, 2009. CXCL10 and autoimmune diseases. Autoimmun Rev., 8: 379-383. PMID: 19105984

Lit, L.C., C.K. Wong, L.S. Tam, E.K. Li and C.W. Lam, 2006. Raised plasma concentration and ex vivo production of inflammatory chemokines in patients with systemic lupus erythematosus. Ann. Rheum. Dis., 65: 209-215. PMID: 15975968

Liu, T.F. and B.M. Jones, 1998. Impaired production of IL-12 in systemic lupus erythematosus. I. Excessive production of IL-10 suppresses production of IL-12 by monocytes. Cytokine, 10: 140-147. PMID: 9512904

Llorente, L., Y. Richaud-Patin, R. Fior, J. AlcocerVarela and J. Wijdenes et al., 1994. In vivo production of interleukin-10 by non-T cells in rheumatoid arthritis, Sjogren's syndrome and systemic lupus erythematosus. A potential mechanism of B lymphocyte hyperactivity and autoimmunity. Arthritis Rheum., 37: 1647-1655. PMID: 7980676

Mellor-Pita, S., M.J. Citores, R. Castejon, M. YebraBango and P. Tutor-Ureta et al., 2009. Monocytes and $\mathrm{T}$ lymphocytes contribute to a predominance of interleukin 6 and interleukin 10 in systemic lupus erythematosus. Cytometry B Clin. Cytom., 76: 261-270. PMID: 19199277

Park, Y.B., S.K. Lee, D.S. Kim, J. Lee and C.H. Lee et al., 1998. Elevated interleukin-10 levels correlated with disease activity in systemic lupus erythematosus. Clin. Exp. Rheumatol., 16: 283288. PMID: 9631750

Premack, B.A. and T.J. Schall, 1996. Chemokine receptors: Gateways to inflammation and infection. Nat Med., 2: 1174-1178. PMID: 8898734

Rousset, F., E. Garcia, T. Defrance, C. Peronne and N. Vezzio et al., 1992. Interleukin 10 is a potent growth and differentiation factor for activated human B lymphocytes. Proc. Natl. Acad. Sci. USA., 89: 1890-1893. PMID: 1371884

Sallusto, F. and A. Lanzavecchia, 2000. Understanding dendritic cell and T-lymphocyte traffic through the analysis of chemokine receptor expression. Immunol. Rev., 177: 134-140. PMID: 11138771 
Sallusto, F., 1999. The role of chemokines and chemokine receptors in $\mathrm{T}$ cell priming and Th1/Th2-mediated responses. Haematologica, 84: 28-31. PMID: 10907461

Santer, D.M., T. Yoshio, S.Minota, T. Moller and K.B. Elkon, 2009. Potent induction of IFN-alpha and chemokines by autoantibodies in the cerebrospinal fluid of patients with neuropsychiatric lupus. J. Immunol., 182: 1192-1201. PMID: 19124763

Shah, D., R. Kiran, A. Wanchu and A. Bhatnagar, 2010. Oxidative stress in systemic lupus erythematosus: relationship to Th1 cytokine and disease activity. Immunol. Lett., 129: 7-12. PMID: 20105444

Shimizu, S., H. Nakashima, K. Karube, K. Ohshima and K. Egashira, 2005. Monocyte chemoattractant protein-1 activates a regional Th1 immunoresponse in nephritis of MRL/lpr mice. Clin. Exp. Rheumatol., 23: 239-242. PMID: 15895897

Shiroiwa, W., K. Tsukamoto, M. Ohtsuji, Q. Lin and A. Ida et al., 2007. IL-4Ralpha polymorphism in regulation of IL-4 synthesis by T cells: Implication in susceptibility to a subset of murine lupus. Int. Immunol., 19: 175-183. PMID: 17189592

Tokano, Y., S. Morimoto, H. Kaneko, H. Amano and K. Nozawa et al., 1999. Levels of IL-12 in the sera of patients with Systemic Lupus Erythematosus (SLE)--relation to Th1- and Th2-derived cytokines. Clin. Exp. Immunol., 116: 169-173. PMID: 10209522

Tucci, M., L. Lombardi, H.B. Richards, F. Dammacco and F. Silvestris, 2008. Overexpression of interleukin-12 and $\mathrm{T}$ helper 1 predominance in lupus nephritis. Clin. Exp. Immunol., 154: 247254. PMID: 18795942
Vielhauer, V., H.J. Anders and D. Schlondorff, 2007. Chemokines and chemokine receptors as therapeutic targets in lupus nephritis. Semin. Nephrol., 27: 81-97. PMID: 17336691

Vila, L.M., M.J. Molina, A.M. Mayor, J.J. Cruz and E. Rios-Olivares et al., 2007. Association of serum MIP-1alpha, MIP-1beta and RANTES with clinical manifestations, disease activity and damage accrual in systemic lupus erythematosus. Clin. Rheumatol., 26: 718-722. PMID: 16924394

Wang, Y., S. Ito, Y. Chino, D. Goto and I. Matsumoto et al., 2010. Laser microdissection-based analysis of cytokine balance in the kidneys of patients with lupus nephritis. Clin. Exp. Immunol., 159: 1-10. PMID: 19807734

Wong, C.K., C.Y. Ho, E.K. Li and C.W. Lam, 2000. Elevation of proinflammatory cytokine (IL-18, IL17, IL-12) and Th2 cytokine (IL-4) concentrations in patients with systemic lupus erythematosus. Lupus, 9: 589-593. PMID: 11035433

Youinou, P. and C. Jamin, 2009. The weight of interleukin-6 in B cell-related autoimmune disorders. J. Autoimmun, 32: 206-210. PMID: 19307104

Zlotnik, A. and O. Yoshie, 2000. Chemokines: A new classification system and their role in immunity. Immunity, 12: 121-127. PMID: 10714678 